\title{
Slow light through tightly-coupled light waves and acoustic waves in nanoscale waveguides
}

\author{
Zheng Wang, The Univ. of Texas at Austin (USA); Wenjun Qiu, Massachusetts Institute of \\ Technology (USA); Peter Rakich, Yale Univ. (USA); Heedeuk Shin, Sandia National Labs. (USA); \\ Hui Dong, The Univ. of Texas at Austin (USA)
}

\begin{abstract}
We develop a general framework of evaluating slow-light performance using Stimulated Brillouin Scattering (SBS) in optical waveguides via the overlap integral between optical and elastic eigen-modes. We show that spatial symmetry of the optical force dictates the selection rules of the excitable elastic modes. By applying this method to a rectangular silicon waveguide, we demonstrate the spatial distributions of optical force and elastic eigen-modes jointly determine the magnitude and scaling of SBS gain coefficient in both forward and backward SBS processes. We further apply this method to inter-modal SBS process, and demonstrate that the coupling between distinct optical modes is necessary to excite elastic modes with all possible symmetries.
\end{abstract}

\section{INTRODUCTION}

Stimulated Brillouin Scattering (SBS) is a third order nonlinear process in which two optical modes are coupled through an elastic mode ${ }^{1,2}$. In a waveguide system, the interference of pump and Stokes waves generates a time-varying optical force at the beat frequency. The optical force, while at resonance with an elastic mode at the phase-matching wavevector, excites the mechanical vibration of the waveguide, which can in turn scatter light between the pump and Stokes waves. Since its discovery, SBS has been extensively studied with a variety of applications in efficient phonon generation ${ }^{[3,4}$, optical frequency conversion ${ }^{5-7}$, slow light ${ }^{8-11}$, and signal processing techniques ${ }^{12,13}$.

The optical force that mediates SBS includes electrostriction force and radiation pressure ${ }^{14,15}$. Electrostriction is an intrinsic material nonlinearity, which arises from the tendency of materials to become compressed in the region of high optical intensity. In previous studies, electrostriction is treated as a bulk nonlinearity with only electrostriction body force taken into account ${ }^{1,2}$. We find that the discontinuities of optical intensities and photoelastic constants can generate electrostriction pressure on material boundaries. Radiation pressure is another boundary nonlinearity, which arises from the interaction of light with the material boundaries with discontinuous dielectric constant ${ }^{16,17}$. For nanoscale structures, radiation pressure is radically enhanced, enabling a variety of optomechanics applications ${ }^{18-23}$. Within nanoscale waveguides, the distributions of electrostriction force and radiation pressure are quite different. The interplay between these two effects creates new degree of freedoms of tailoring SBS process.

In translationally invariant waveguides, SBS can be categorized into forward SBS (FSBS) and backward SBS (BSBS). In FSBS, the pump and Stokes waves propagate in the same direction, generating translationally invariant optical forces, which excite standing elastic modes ${ }^{6}$. In BSBS, the pump and Stokes waves propagate in the counter directions, generating translationally varying optical forces, which excite traveling elastic modes. SBS can also occur between distinct optical modes ${ }^{24-28}$. Intermode SBS has been used in optical signal isolation and Brillouin cooling of mechanical devices.

The strength of SBS nonlinearity is characterized by the SBS gain. There have been many theoretical studies on calculating SBS gain through some forms of overlap integral between optical and elastic eigen-modes ${ }^{1,2,6,24,27-31}$. These treatments, while accurate for microscale waveguides, suffer from two drawbacks for nanoscale waveguides. First, most previous treatments are based on nonlinear polarization current. The calculated SBS gain only captures electrostriction body forces, but doesn't account for boundary nonlinearities such as electrostriction pressure and radiation pressure. This issue becomes significant for nanoscale waveguides where the boundary effect is radically enhanced. Second, some previous studies assume the optical mode is linearly polarized, while some studies describe the elastic modes by the density modulation rather than the displacement vector. For nanoscale waveguides, the vector nature of optical and elastic modes have to be fully evaluated.

Advances in Slow and Fast Light VI, edited by Selim M. Shahriar, Frank A. Narducci, Proc. of SPIE Vol. 8636, 863607 · C 2013 SPIE · CCC code: 0277-786X/13/\$18 · doi: 10.1117/12.2010049 
In this article, we propose a general method of calculating SBS gains via the overlap integral between optical and elastic eigen-modes. Within this formalism, all kinds of optical forces are taken into account, with bulk and boundary nonlinearities formulated as bulk and boundary integrals over the waveguide cross-section. In addition, both the optical and elastic modes are treated as vector fields, allowing for the most general forms of dielectric and elastic tensors. Armed with this formalism, we study the SBS process of a silicon rectangular waveguide. We will show that all the optical forces in FSBS are transverse, and the constructive combination of electrostriction force and radiation pressure can generate large FSBS gain for certain elastic modes. In contrast, the optical force in BSBS is largely longitudinal, and the maximal BSBS gain among all the elastic modes approaches the conventional BSBS gain. We further apply this formalism to intermode SBS. By coupling optical modes with distinct symmetries, optical forces with all possible symmetries can be generated, and the elastic modes with the same symmetry can be excited. Finally, we study the FSBS process when the silicon waveguide is put on top of a silica substrate. Both the frequency response calculation and leaky eigen-mode analysis reveal that the coupling to the radiative modes of the substrate significantly introduces a large radiative loss which significantly reduces the SBS gain of the structure.

\section{CALCULATING THE SBS GAIN VIA OVERLAP INTEGRAL}

To start with, we develop a general framework of calculating the SBS gain. Consider a translationally invariant waveguide in $x$ direction. In a typical SBS process, the pump wave $\mathbf{E}_{p} e^{i(k p x-\omega p t)}$ and the Stokes wave $\mathbf{E}_{s} e^{i(k s x-\omega s t)}$ generate optical forces at the phase-matching wavevector $q=k_{p}-k_{s}$ and the beat frequency $\Omega=\omega_{p}-\omega_{s}$. This optical force can excite mechanical vibrations which enables the parametric conversion between pump and Stokes waves. This process can be describe by the following relation ${ }^{1}$ :

$$
\frac{d P_{s}}{d x}=g P_{p} P_{s}-\alpha_{s} P_{s}
$$

Here, $P_{p}$ and $P_{s}$ are the guided power of the pump and Stokes waves, and $g$ is the SBS gain. Through particle flux conservation, SBS gain is given by the following formula ${ }^{15}$ :

$$
\mathrm{g}(\Omega)=\frac{\omega_{s}}{2 \Omega P_{p} P_{s}} \mathfrak{R}\left\langle\mathbf{f}, \frac{d \mathbf{u}}{d t}\right\rangle
$$

where $\mathbf{f}$ is the optical force generated by pump and Stokes waves, and $\mathbf{u}$ is the elastic response of the waveguide induced by $\mathbf{f}$. The overlap integral is defined over the waveguide cross-section. The optical power of a waveguide is given by $P=v_{g}\langle\mathbf{E}, \epsilon \mathbf{E}\rangle / 2$, where $v_{g}$ is the optical group velocity.

Under normalization conditions $\left\langle\mathbf{E}_{\mathrm{p}}, \epsilon \mathbf{E}_{\mathrm{p}}\right\rangle=1$ and $\left\langle\mathbf{E}_{\mathrm{s}}, \epsilon \mathbf{E}_{\mathrm{s}}\right\rangle=1$, we have

$$
\mathrm{g}(\Omega)=\frac{2 \omega_{s}}{v_{g p} v_{g s}} \mathfrak{J}\langle\mathbf{f}, \mathbf{u}\rangle
$$

To further simply (3), we have to consider the equation governing the elastic response $\mathbf{u} e^{-i \Omega t}$ under external forces $\mathbf{f} e^{-i \Omega t}$. When elastic loss are ignored, we have ${ }^{32}$ :

$$
-\rho \Omega^{2} u_{i}=\frac{\partial}{\partial x_{j}} c_{i j k l} \frac{\partial u_{l}}{\partial x_{k}}+f_{i}
$$

where $\rho$ is the mass density, and $c_{i j k l}$ is the elastic tensor. $c_{i j k l}$ has two important properties. First, it is symmetric about the first two and last two indies: $c_{i j k l}=c_{i k k l}, c_{i j l k}=c_{i j k l}$. Second, the interchange of the first two indies and the last two does not affect the value of $c_{i j k l}: c_{k l i j}=c_{i j k l} 32$. Without $\mathbf{f}$, the equation above is the elastic eigen-equation. Using the symmetry properties of $c_{i j k l}$, we can show that the operator in the eigen-equation is Hermitian, and the eigen-mode $\mathbf{u}_{m} e^{-i \Omega m}$ satisfies orthonormal condition:

$$
h \mathbf{u}_{m}, \rho \mathbf{u}_{n} \mathrm{i}=\delta_{m n}
$$

When $\mathbf{f}$ is present, we can decompose $\mathbf{u}$ into the set of eigen-modes $\mathbf{u}=\mathrm{P}_{m} b_{m} \mathbf{u}_{m}$. Using the orthonormal condition, we have:

$$
b_{m}=\frac{\left\langle\mathbf{u}_{m}, \mathbf{f}\right\rangle}{\Omega_{m}^{2}-\Omega^{2}}
$$


Now we add elastic loss to this system. The commonly encountered elastic loss mechanism is air damping, thermoelastic dissipation, and clamping losses ${ }^{33}$. The first order effect of loss can be captured by changing $\Omega_{m}$ to $\Omega_{m}-i \Gamma_{m} / 2$. Assuming quality factor $Q_{m}=\Omega_{m} / \Gamma_{m}$ is well above 1, we have,

$$
b_{m}=\frac{\left\langle\mathbf{u}_{m}, \mathbf{f}\right\rangle}{\Omega_{m} \Gamma_{m}} \frac{\Gamma_{m} / 2}{\Omega_{m}-\Omega-i \Gamma_{m} / 2}
$$

Inserting (7) into (3), we can see that the total SBS gain is the sum of SBS gains of individual elastic modes.

$$
g(\Omega)=\sum_{m} G_{m} \frac{\left(\Gamma_{m} / 2\right)^{2}}{\left(\Omega-\Omega_{m}\right)^{2}+\left(\Gamma_{m} / 2\right)^{2}}
$$

The SBS gain of a single elastic mode has a Lorentian shape and a peak value:

$$
G_{m}=\frac{2 \omega Q_{m}}{\Omega_{m}^{2} v_{g p} v_{g s}}\left|\left\langle\mathbf{f}, \mathbf{u}_{\mathrm{m}}\right\rangle\right|^{2}
$$

where we have used the fact that $\Omega \ll \omega_{p}, \omega_{s}$ and $\omega_{p} \approx \omega_{s}=\omega$.

(9) provides a general method to calculate the SBS gain of a waveguide with arbitrary cross section. Specifically, we can use finite element method to solve for the pump and Stokes optical modes at a given $\omega$ and the elastic modes at the phase-matching wavevector $q=k_{p}-k_{s}$. The SBS of each elastic mode can be calculated by taking the overlap integral between optical forces and elastic displacement. Here, body forces are integrated over the waveguide cross-section, while pressures are integrated over the waveguide edge. In addition, (9) shows that the SBS gain is determined by the frequency ratio, the elastic loss factor, the optical group velocities, and the overlap integral between optical forces and elastic eigen-modes. Moreover, (9) provides a way to separate the effects of various optical forces. Specifically, the overlap integral is the sum of all optical forces:

$$
\left\langle\mathbf{f}, \mathbf{u}_{\mathrm{m}}\right\rangle=\sum_{n}\left\langle\mathbf{f}_{\mathrm{n}}, \mathbf{u}_{\mathrm{m}}\right\rangle
$$

The amplitudes and relative phases of individual overlap integrals determine the separated contributions and interference effects of various optical forces.

A key step of applying (9) is to calculate optical forces from pump and Stokes waves. Throughout the study, we consider electrostriction force and radiation pressure. Electrostriction forces are derived from electrostriction tensor. The instantaneous electrostriction tensor is given by:

$$
\sigma_{i j}^{E S}=-\frac{1}{2} \epsilon_{o} n^{4} p_{i j k l} E_{k} E_{l}
$$

where $n$ is the refractive index, and $p_{i j k l}$ is the photoelastic tensor ${ }^{34}$. In a waveguide system, the total electric field is given by $\left(\mathbf{E}_{p} e^{i(k p x-\omega p t)}+\mathbf{E}_{s} e^{i(k s x-\omega s t)}\right) / 2+$ c.c. Inserting this expression to (11), and filtering out the components with frequency $\Omega$, we get the time-harmonic electrostriction tensor $\sigma i j e i(q x-\Omega t)$ :

$$
\sigma_{i j}=-\frac{1}{4} \epsilon_{o} n^{4} p_{i j k l}\left(E_{p k} E_{s l}^{*}+E_{p l} E_{s k}^{*}\right)
$$

For simplicity, we assume that the crystal structure of the waveguide material is symmetric about $x=0, y=0$, and $z=0$. Therefore, $p_{i j k l}$ is zero if it contains odd number of a certain component. In compact notation, (12) can be written as:

$$
\left[\begin{array}{c}
\sigma_{x x} \\
\sigma_{y y} \\
\sigma_{z z} \\
\sigma_{y z} \\
\sigma_{x z} \\
\sigma_{x y}
\end{array}\right]=-\frac{1}{2} \epsilon_{o} n^{4}\left[\begin{array}{llllll}
p_{11} & p_{12} & p_{13} & & & \\
p_{12} & p_{22} & p_{23} & & & \\
p_{13} & p_{23} & p_{33} & & & \\
& & & p_{44} & & \\
& & & & p_{55} & \\
& & & & & p_{66}
\end{array}\right]\left[\begin{array}{c}
E_{p x} E_{s x}^{*} \\
E_{p y} E_{s y}^{*} \\
E_{p z} E_{s z}^{*} \\
E_{p y} E_{s z}^{*}+E_{p z} E_{s y}^{*} \\
E_{p x} E_{s z}^{*}+E_{p z} E_{s x}^{*} \\
E_{p x} E_{s y}^{*}+E_{p y} E_{s x}^{*}
\end{array}\right]
$$

Electrostriction force is given by the divergence of electrostriction tensor. In a system consisting of homogeneous materials, electrostriction forces can exist inside each material (electrostriction body force) and on the interfaces (electrostriction pressure). Electrostriction body force is $\mathbf{f}^{E S} e^{i(q x-\Omega t)}$ : 


$$
\begin{aligned}
& f_{x}^{E S}=-i q \sigma_{x x}-\partial_{y} \sigma_{x y}-\partial_{z} \sigma_{x z} \\
& f_{y}^{E S}=-i q \sigma_{x y}-\partial_{y} \sigma_{y y}-\partial_{z} \sigma_{y z} \\
& f_{z}^{E S}=-i q \sigma_{x z}-\partial_{y} \sigma_{z y}-\partial_{z} \sigma_{z z}
\end{aligned}
$$

Electrostriction pressure on the interface between material 1 and 2 is given by $\mathbf{F}^{E S} e^{i(q x-\Omega t)}$ ( normal vector $n$ points from 1 to 2):

$$
F_{i}^{E S}=\left(\sigma_{1 i j}-\sigma_{2 i j}\right) n_{j}
$$

Under a particular phase, the optical mode of the waveguide $\mathbf{E} e^{i(k x-\omega t)}$ have imaginary $E_{x}$ and real

$E_{y, z}$. From (13), we can see that $\sigma_{x x}, \sigma_{y y}, \sigma_{z z}$, and $\sigma_{y z}$ are real while $\sigma_{x y}$ and $\sigma_{x z}$ are imaginary. From (14) and (15), we can see that for both electrostriction body force and electrostriction pressure, the transverse component is real while the longitudinal component is imaginary.

Radiation pressure is derived from Maxwell Stress Tensor (MST). For a dielectric system $(\mu=1)$ without free charges $(\rho$ $=0, J=0$ ), radiation pressure is localized where the gradient of is nonzero ${ }^{35,36}$. For a system consisting of homogeneous materials, radiation pressure only exists on the interfaces. The dielectric part of instantaneous MST is:

$$
\mathrm{T}_{i j}=\epsilon_{o} \epsilon\left(E_{i} E_{j}-\frac{1}{2} \delta_{i j} E^{2}\right)
$$

The instantaneous pressure on the interface between material 1 and 2 is:

$$
F_{i}^{R P}=\left(T_{2 i j}-T_{1 i j}\right) n_{j}
$$

By decomposing the electric field into its normal and tangent components $\mathbf{E}=E_{n} \mathbf{n}+E_{t} \mathbf{t}$, and using the boundary condition $\epsilon_{1} E_{1 n}=\epsilon_{2} E_{2 n}=D_{n}$ and $E_{1 t}=E_{2 t}=E_{t}$, we can show that:

$$
\mathbf{F}^{\mathrm{RP}}=-\frac{1}{2} \epsilon_{o} E_{t}^{2}\left(\epsilon_{2}-\epsilon_{1}\right) \mathbf{n}+\frac{1}{2} \epsilon_{o}^{-1} D_{n}^{2}\left(\epsilon_{2}^{-1}-\epsilon_{1}^{-1}\right) \mathbf{n}
$$

Inserting the total electric field $\left(\mathbf{E}_{p} e^{i(k p x-\omega p t)}+\mathbf{E}_{s} e^{i(k s x-\omega s t)}\right) / 2+c . c$ to the expression above, and filtering out the components with frequency $\Omega$, we can get the time-harmonic radiation pressure $\mathbf{F} R P \operatorname{ei}(q x-\Omega t)$ :

$$
\mathbf{F}^{\mathrm{RP}}=-\frac{1}{2} \epsilon_{o} E_{p t} E_{s t}^{*}\left(\epsilon_{2}-\epsilon_{1}\right) \mathbf{n}+\frac{1}{2} \epsilon_{o}^{-1} D_{p n} D_{s n}^{*}\left(\epsilon_{2}^{-1}-\epsilon_{1}^{-1}\right) \mathbf{n}
$$

(19) shows that radiation pressure is always in the normal direction. For translationally invariant waveguide, this also means radiation pressure is transverse and real.

Combining (9) with the calculation of optical forces, we are ready to explore the SBS nonlinearity of nanoscale waveguides. Before that, it is instructive to compare (9) with the conventional BSBS gain ${ }^{2}$. We can show that (9) converges to the conventional BSBS gain under plane-wave approximations for both optical and elastic modes. Specifically, consider the coupling between two counter propagating optical plane-waves through an elastic plane-wave. The optical plane-wave is linearly polarized in $y$ direction. The elastic plane-wave is pure longitudinal traveling at velocity $V_{L}$. Under this setup, nonzero optical forces include the longitudinal electrostriction body force, and the transverse components of electrostriction pressure and radiation pressure. Only the longitudinal electrostriction body force contributes nonzero overlap integral:

$$
f_{x}^{E S}=-i q \sigma_{x x}=\frac{1}{2} i q \epsilon_{o} n^{4} p_{12} E_{y}^{2}
$$

Inserting this expression into (9), and using the fact that $\Omega=q V_{L}$ and $q=2 k$, we can show that:

$$
G_{o}=\frac{\omega^{2} n^{7} p_{12}^{2}}{c^{3} \rho V_{L} \Gamma} \frac{1}{A}
$$

where $A$ is the cross-sectional area of the waveguide. This is exactly the conventional BSBS gain. For microscale waveguides, the plane-wave approximation is valid, and (9) converges to $G_{0}$. For nanoscale waveguides, (9) can deviate from $G_{0}$ significantly because of the vector nature of optical and elastic modes as well as the enhanced boundary nonlinearities. 


\section{SILICON RECTANGULAR WAVEGUIDE: INTRAMODE COUPLING}

In this section, we apply the general formalism to study the SBS process of a silicon rectangular waveguide suspended in air (Figure 1 insert). The cross-section is $a$ by $0.9 a$. For silicon, we use refractive index $n=3.5$, Young's modulus $E=$ $170 \times 10^{9} \mathrm{~Pa}$, Poisson's ratio $v=0.28$, and density $\rho=2329 \mathrm{~kg} / \mathrm{m}^{2}$. In addition, we assume that the [100], [010], and [001] symmetry direction of this crystalline silicon coincide with the $x, y$, and $z$ axis respectively. Under this orientation, the photo-elastic tensor $p_{i j k l}$ in contracted notation is $\left[p_{11}, p_{12}, p_{44}\right]=[-0.09,0.017,-0.051]^{37}$. The structure has two symmetry plane $y=0$ and $z=0$. Both optical modes and elastic modes have fixed parities about these planes.

The fundamental optical modes are $E_{y 11}$ and $E_{z 11}$ (Figure 1(a)). $E_{y 11}$ is even about $z=0$ and odd about $y=0$ with a large $E_{y}$ component. $E_{z 11}$ has the opposite parities and slightly higher frequencies. Throughout the study, we assume the pump wavelength is $1.55 \mu \mathrm{m}$. So $a$ is the product of $1.55 \mu \mathrm{m}$ and $\omega$ in unit of $2 \pi c / a$, and different operating point in the dispersion diagram corresponds to different $a$. For intramode coupling, we assume that pump and Stokes waves come from $E_{y 11}$. Since $\Omega / \omega \approx V_{L} / c$ is on the order of $10^{-4}$, pump and Stokes waves approximately corresponds to the same mode $\mathbf{E} e^{i(k x-\omega t)}$. The optical force induced by intramode coupling is always symmetric about both $y=0$ and $z=0$. We only consider elastic modes with the same parities (Figure 2(b)). Here, E-modes are the actual eigen-modes, while Pmodes (S-modes) are the constrained eigen-modes with pure longitudinal (transverse) displacement. At $q=0$, the geometric symmetry plane $x=0$ is recovered. Elastic modes that are odd (even) about this plane only have longitudinal (transverse) movement. Therefore, elastic modes at $q=0$ are pure P-modes or S-modes (Figure 1(c)). Elastic modes at nonzero $q$ are hybrid. Similar to the optical mode, we can choose a proper phase so that $u_{x}$ is imaginary while $u_{y, z}$ are real.

\subsection{Forward SBS}

In FSBS, $\mathbf{E}_{p}=\mathbf{E}_{s}=\mathbf{E}$ and $q=0$. (13) can be simplified to:

$$
\left[\begin{array}{c}
\sigma_{x x} \\
\sigma_{y y} \\
\sigma_{z z} \\
\sigma_{y z} \\
\sigma_{x z} \\
\sigma_{x y}
\end{array}\right]=-\frac{1}{2} \epsilon_{o} n^{4}\left[\begin{array}{cccccc}
p_{11} & p_{12} & p_{13} & & & \\
p_{12} & p_{22} & p_{23} & & & \\
p_{13} & p_{23} & p_{33} & & & \\
& & & p_{44} & & \\
& & & & p_{55} & \\
& & & & & p_{66}
\end{array}\right]\left[\begin{array}{c}
\left|E_{x}\right|^{2} \\
\left|E_{y}\right|^{2} \\
\left|E_{z}\right|^{2} \\
2 \Re\left(E_{y} E_{z}^{*}\right) \\
0 \\
0
\end{array}\right]
$$

Therefore, $\sigma_{x y}=\sigma_{x z}=0$. From (14) and (15), we can see that $f_{x}^{E S}=F_{x}^{E S}=0$. So both electrostriction force and radiation pressure in FSBS are transverse. We pick an operating point at $\omega=0.203(2 \pi c / a), k=0.75(\pi / a)$ with $a=315 \mathrm{~nm}$, and compute the force distribution ( Figure 2(a)). Electrostriction body force is largely in $y$ direction. This is because $E_{y}$ is the dominant component in electric field and $\left|p_{11}\right|$ is about five times larger than $\left|p_{12}\right|$. Electrostriction pressure points inwards. Radiation pressure points outwards. Radiation pressure is about five times larger than electrostriction pressure. The transverse nature of optical force combined with the fact that elastic modes are either P-modes or S-modes at $q=0$ indicates that only S-modes have nonzero FSBS gains. We compute the corresponding FSBS gains using a quality factor $Q=1000$ for all the elastic modes (Figure 2(b)). As expected, only S-modes E2, E3, and E5 have nonzero gains. E2 has the largest gain of $1.72 \times 10^{4} \mathrm{~m}^{-1} \mathrm{~W}^{-1}$, which comes from a constructive combination of electrostriction force $\left(0.42 \times 10^{4} \mathrm{~m}^{-}\right.$ $\left.{ }^{1} \mathrm{~W}^{-1}\right)$ and radiation pressure $\left(0.44 \times 10^{4} \mathrm{~m}^{-1} \mathrm{~W}^{-1}\right)$. E5 has a total gain of $0.51 \times 10^{4} \mathrm{~m}^{-1} \mathrm{~W}^{-1}$, which mainly comes from radiation pressure $\left(1.72 \times 10^{4} \mathrm{~m}^{-1} \mathrm{~W}^{-1}\right)$.

Next, we vary $a$ from $250 \mathrm{~nm}$ to $2.5 \mu \mathrm{m}$ by lifting the operating point at the optical dispersion diagram from $0.16(2 \pi c / a)$ to $1.61(2 \pi c / a)$ (Figure 2(c)). For both E2 and E5, electrostriction FSBS gain scales as $1 / a^{2}$ for large $a$. This can be understood by a detailed analysis of (9). Under normalization condition $\langle\mathbf{E}, \epsilon \mathbf{E}\rangle=1$, the electrostriction tensor scales as $1 / a^{2}$. Since electrostriction force is essentially the divergence of electrostriction tensor, the total electrostriction force that apply to the right half of the waveguide scales as $1 / a^{3}$. Under normalization condition hu, $\rho \mathbf{u i}=1$, u scales as $1 / a$. So the overlap integral scales as $1 / a^{2}$. Under a fixed $Q$, the electrostriction FSBS gain scales as $1 / a^{2}$. The radiation pressure FSBS gain scales as $1 / a^{8}$ for E2 and $1 / a^{6}$ for E5. This can also be understood from a breakdown of (9). Given the input power, the sum of average pressure on the top and side boundaries of the rectangular waveguide is proportional to $\left(n_{g}-\right.$ $\left.n_{p}\right) / A$, where $n_{g}\left(n_{p}\right)$ is the group (phase) index, and $A$ is the waveguide cross-section. When the waveguide is scaled up with a fixed aspect ratio, $n_{g}-n_{p}$ shrinks in the order of $1 / A$. So the sum of average radiation pressure scales as $1 / a^{4}$, and the radiation pressure FSBS gains should scales as $1 / a^{6}$. For E2, the radiation pressure on the side and top boundaries 
have opposite effects. So it is the difference rather than the sum between side and top radiation pressures that determines the overlap integral. This is why the radiation pressure FSBS gain of E2 decays faster.

\subsection{Backward SBS}

In BSBS, $\mathbf{E}_{p}=\mathbf{E}, \mathbf{E}_{s}=\mathbf{E}^{*}$, and $q=2 k$. (13) can be simplified to:

$$
\left[\begin{array}{l}
\sigma_{x x} \\
\sigma_{y y} \\
\sigma_{z z} \\
\sigma_{y z} \\
\sigma_{x z} \\
\sigma_{x y}
\end{array}\right]=-\frac{1}{2} \epsilon_{o} n^{4}\left[\begin{array}{cccccc}
p_{11} & p_{12} & p_{13} & & & \\
p_{12} & p_{22} & p_{23} & & & \\
p_{13} & p_{23} & p_{33} & & & \\
& & & p_{44} & & \\
& & & & p_{55} & \\
& & & & & p_{66}
\end{array}\right]\left[\begin{array}{c}
E_{x}{ }^{2} \\
E_{y}{ }^{2} \\
E_{z}{ }^{2} \\
2 E_{y} E_{z} \\
2 E_{x} E_{z} \\
2 E_{x} E_{y}
\end{array}\right]
$$

All components of $\sigma_{i j}$ are nonzero, generating electrostriction force with both longitudinal and transverse components. We pick an operating point at $\omega=0.203(2 \pi c / a), k=0.75(\pi / a)$ with $a=315 \mathrm{~nm}$, and compute the force distribution (Figure 3(a)). Electrostriction body force has large longitudinal component over the waveguide cross-section, which mainly comes from the $-i q \sigma_{x x}$ term in $f_{x}^{E S}$. The hybrid nature of optical forces combined with the fact that all elastic modes are hybrid at nonzero $q$ indicates that all elastic modes have nonzero gains. We compute the corresponding BSBS gains using a quality factor $Q=1000$ for all the elastic modes (Figure 3(b)). For E1 and E2, electrostriction force and radiation pressure add up destructively, resulting small BSBS gains of $0.089 \times 10^{4} \mathrm{~m}^{-1} \mathrm{~W}^{-1}$ and $0.089 \times 10^{4} \mathrm{~m}^{-1} \mathrm{~W}^{-1}$ respectively.

Next, we vary $a$ from $250 \mathrm{~nm}$ to $2.5 \mu \mathrm{m}$ and compute the corresponding BSBS gains for E1 and the conventional BSBS gain $G_{0}$ (Figure 2(c)). The electrostriction BSBS gain of E1 decays very fast. In contrast, $G_{0}$ scales as $1 / a^{2}$ as indicated by (21). This difference comes from the fact that the conventional BSBS corresponds to the longitudinal plane wave, but E1 quickly deviates from longitudinal plane wave as the dimensionless $q$ increases. There are two ways to recover the conventional BSBS gain from (9). First, we consider the constrained longitudinal modes ( P-modes ) in Figure 1(b). P1 is just the longitudinal plane wave. The computed electrostriction BSBS for P1 converges to $G_{0}$ as $a$ increases (Figure 3(c)). Second, the dispersion curve of P1 pass through different E-modes as the dimensionless $q$ increases. The E-modes at the intersection points with P1 become P1-like with large longitudinal movement over the waveguide cross-section. These modes should have a large electrostriction BSBS gain close to that of P1. Therefore, we compute the electrostriction BSBS gain for E1 through E500 for different $a$ (Figure 3(c)). The maximal gain among all the modes converges to $G_{0}$ as expected.

\section{SILICON RECTANGULAR WAVEGUIDE: INTERMODE COUPLING}

In this section, we apply our formalism to intermode coupling of the same silicon rectangular waveguide. For intramode coupling, the optical force is always symmetric about $y=0$ and $z=0$, exciting elastic modes with the same parities (Emodes). For intermode coupling, however, optical forces with all possible symmetries can be generated, and elastic modes with all possible symmetries can be excited. For instance, we consider the coupling between $E_{y 11}$ (pump) and $E_{z 11}$ ( Stokes ). The operating point is $\omega=0.203(2 \pi c / a), k_{p}=0.750(\pi / a), k_{s}=0.665(\pi / a)$, and $q=0.085(\pi / a)$ with $a=315 \mathrm{~nm}$. Because $E_{y 11}$ and $E_{z 11}$ have the opposite symmetries about both $y=0$ and $z=0$, the induced optical force is antisymmetric about both planes (Figure 4(a)). Both electrostriction body force and radiation pressure try to pull the waveguide in one diagonal and push the waveguide in the other diagonal. Electrostriction pressure has the opposite effect, but is much weaker than the radiation pressure.

Under such optical force, elastic modes which are odd about both $y=0$ and $z=0$ (O-modes) can be excited. We calculated the SBS gains of O1 through O5 using a quality factor $Q=1000$ for all the modes (Figure 4(b)). O1 represents rotation about $x$ axis. So the overlap integral is proportional to the torque. The $y$ component and $z$ component of the optical forces generate torques with opposite signs, which reduce the overlap integral. It is the small elastic frequency $(\Omega$ $\left.=0.024\left(2 \pi V_{L} / a\right)\right)$ that generates a significant SBS gain. O2 represents a breathing motion along the diagonal, which coincides with the optical force distribution quite well. The constructive combination between electrostriction force and 
radiation pressure results in total gain of $1.54 \times 10^{4} \mathrm{~m}^{-1} \mathrm{~W}^{-1}$. O3 only have small gains since it is dominantly longitudinal while the optical forces are dominantly transverse. The SBS gains of O4, O5 and higher order modes are close to zero because the overlap integrals are largely canceled out by the complicated modal profiles.

\section{SUBSTRATE EFFECT}

In the discussion above, the silicon waveguide is suspended in air, which provides tight optical guidance and nearly perfect elastic guidance because of the huge elastic impedance mismatch between silicon and air. When the waveguide is put on top of a substrate with smaller refractive index, the optical guidance is still good, but the elastic guidance needs to be carefully examined. For BSBS, $q=2 k$ is not zero. If the phonon travels faster in the substrate than the waveguide, traveling elastic mode that is localized around the waveguide can be formed, enabling the on-chip realization of BSBS process ${ }^{7]}$. For FSBS, $q$ is close to zero, and elastic modes travel transversely. No matter whether phonon travels faster or slower in the substrate, the transverse vibration of the waveguide will always excite the radiative modes of the substrate. Therefore, the elastic modes in the waveguide become leaky modes. The quality factor of these modes can be written as $Q_{t o t}=\left(Q_{a b s}{ }^{-1}+Q_{r a d}{ }^{-1}\right)^{-1}$, where $Q_{a b s}$ corresponds to the dissipative losses, and $Q_{\text {rad }}$ corresponds to leakage into the substrate. Because of the large contact area and strong coupling between the waveguide and substrate, $Q_{\text {rad }}$ can be quite small, significantly reducing the FSBS gain of the whole structure.

To further illustrate the substrate effect, we put the silicon rectangular waveguide on a silica substrate and calculate the FSBS gains at operating point $\omega=0.201(2 \pi c / a)$ and $k=0.75(\pi / a)$ with $a=311 \mathrm{~nm}$ (Figure 5). For leaky modes with strong coupling to the external channels, the orthonormal condition (5) is not exact. Therefore, we computed the FSBS gains using frequency response formula (3) rather than the eigen-mode formula (9). To model $Q_{a b s}=1000$, we used a bulk loss factor of 0.001 for the silicon waveguide. To model $Q_{\text {rad }}$, we gradually turn on the loss factor of the substrate in the outer region. The calculated FSBS gain spectrum has two peaks. The leaky modes that give rise to these two peaks are similar to E2 and E5 of the suspended waveguide (Figure 2). However, these leaky modes have much wider bandwidth and much smaller peak FSBS gains. For instance, the leaky mode at $\Omega=0.48\left(2 \pi V_{L} / a\right)$ has $Q_{t o t}=5.6$ and a maximal FSBS gain of $87 \mathrm{~m}^{-1} \mathrm{~W}^{-1}$, both of which are about 20 times smaller than the corresponding values of E2. The modal profiles of these leaky modes clearly demonstrate that the strong coupling to the substrate radiative modes significantly reduce the FSBS nonlinearities.

\section{CONCLUDING REMARKS}

In this article, we propose a general framework of calculating the SBS gain via the overlap integral between optical forces and elastic eigen-modes. Through the study of a silicon waveguide, we demonstrate that our method can be applied to both FSBS and BSBS, both intramode coupling and intermode coupling, both nanoscale waveguides and microscale waveguides. Our numerical calculations show that SBS nonlinearity is tightly connected to the symmetry, polarization, and spatial distributions of both optical and elastic modes, which can be further tailored and optimized by material selection and structural design.

\section{REFERENCES}

[1] Boyd, R., [Nonlinear Optics], Academic Press, (2009).

[2] Agrawal, G., [Nonlinear Fiber Optics], Academic Press, (2006).

[3] Chiao, R. Y., Townes, C. H. and Stoicheff, B. P., "Stimulated brillouin scattering and coherent generation of intense hypersonic waves," Phys. Rev. Lett., 12, 592-595(1964).

[4] Dainese, P., Russell, P., Joly, N., Knight, J., Wiederhecker, G., Fragnito, H. , Laude, V. and Khelif, A., "Stimulated brillouin scattering from multi-ghz-guided acoustic phonons in nanostructured photonic crystal fibres," Nature Phys., 2, 388-392(2006).

[5] Kobyakov, A., Sauer, M. and Chowdhury, D., "Stimulated brillouin scattering in optical fibers," Adv. Opt. Photon., 2,1-59 (2010). 
[6] Kang, M. S., Nazarkin, A., Brenn, A. and Russell, P. St. J., "Tightly trapped acoustic phonons in photonic crystal fibres as highly nonlinear artificial raman oscillators," Nature Phys., 5,276-280 (2009).

[7] Pant, R., Poulton, C. G., Choi, D. Y., Mcfarlane, H., Hile, S., Li, E., Thevenaz, L., Luther-Davies, B., Madden, S. J. and Eggleton, B. J., “Onchip stimulated brillouin scattering," Opt. Express, 19, 8285-8290 (2011).

[8] Song, K. Y., Herr'aez, M. G. and Th'evenaz, L., "Observation of pulse delaying and advancement in optical fibers using stimulated brillouin scattering," Opt. Express, 13, 82-88 (2005).

[9] Song, K. Y., Abedin, K. S., Hotate, K., Herr'aez, M. G. and Th'evenaz, L., "Highly efficient brillouin slow and fast light using as2se3 chalcogenide fiber," Opt. Express, 14,5860-5865 (2006).

[10] Okawachi, Y., Bigelow, M. S., Sharping, J. E., Zhu, Z., Schweinsberg, A., Gauthier, D. J., Boyd, R. W. and Gaeta, A. L., "Tunable all-optical delays via brillouin slow light in an optical fiber," Phys. Rev. Lett., 94, 153902 (2005).

[11] Pant, R., Stenner, M. D., Neifeld, M. A. and Gauthier, D. J., "Optimal pump profile designs for broadband sbs slowlight systems," Opt. Express, 16,2764-2777 (2008).

[12] Tomes, M. and Carmon, T., "Photonic micro-electromechanical systems vibrating at xband (11-ghz) rates," Phys. Rev. Lett., 102,113601 (2009).

[13]Zhu, Z., Gauthier, D. J. and Boyd, R. W., "Stored light in an optical fiber via stimulated brillouin scattering," Science, 318(5857), 1748-1750 (2007).

[14] Rakich, P. T., Davids, P. and Wang, Z., "Tailoring optical forces in waveguides through radiation pressure and electrostrictive forces," Opt. Express, 18,14439-14453(2010).

[15] Rakich, P. T., Reinke, C., Camacho, R., Davids, P. and Wang, Z., "Giant enhancement of stimulated brillouin scattering in the subwavelength limit," Phys. Rev. X, 2,011008 (2012).

[16] Povinelli, M. L., Loncar, M., Ibanescu, M., Smythe, E. J., Johnson, S. G., Capasso, F. and Joannopoulos, J.D., "Evanescent-wave bonding between optical waveguides," Opt. Lett., 30,3042-3044 (2005).

[17] Rakich, P. T., Wang, Z. and Davids, P., "Scaling of optical forces in dielectric waveguides: rigorous connection between radiation pressure and dispersion," Opt. Lett., 36,217-219 (2011).

[18] Kippenberg, T. J. and Vahala, K. J., "Cavity optomechanics: Back-action at the mesoscale," Science, 321(5893), $1172-1176(2008)$.

[19] Eichenfield, M., Michael, C. P., Perahia, R. and Painter, O., "Actuation of micro-optomechanical systems via cavity-enhanced optical dipole forces," Nature Photonics, 1, 416-422 (2007).

[20] Eichenfield, M., Chan, J., Safavi-Naeini, A. H., Vahala, K. J. and Painter, O., "Modeling dispersive coupling andlosses of localized optical andmechanical modes in optomechanicalcrystals," Opt. Express, 17, 20078-20098 (2009).

[21] Rakich, P. T., Popovi, M. A., Soljacic, M. and Ippen, E. P., "Trapping, corralling and spectral bonding of optical resonances through optically induced potentials," Nature Photonics, 1, 658-665 (2007).

[22]Li, M., Pernice, W. H. P. and Tang, H. X., "Tunable bipolar optical interactions between guided lightwaves," Nature Photonics 3, 464-468 (2009).

[23] Roels, J., Vlaminck, I. D., Lagae, L., Maes, B., Thourhout, D. V. and Baets, R., "Tunable optical forces between nanophotonic waveguides," Nature Nanotechnology 4, 510-513 (2009).

[24]Kang, M. S., Brenn, A. and Russell, P. St. J., "All-optical control of gigahertz acoustic resonances by forward stimulated interpolarization scattering in a photonic crystal fiber," Phys. Rev. Lett. 105, 153901 (2010).

[25] Bahl, G., Tomes, M., Marquardt, F. and Carmon, T., "Observation of spontaneous brillouin cooling," Nature Phys. 8, 203-207 (2012).

[26] Yu, Z. and Fan, S., "Complete optical isolation created by indirect interband photonic transitions," Nature Photonics 3, 91-94 (2008).

[27] Huang, X. and Fan, S., "Complete all-optical silica fiber isolator via stimulated brillouin scattering," J. Lightwave Technol. 29, 2267-2275 (2011).

[28] Kang, M. S., Butsch, A. and Russell, P. St. J., "Reconfigurable light-driven opto-acoustic isolators in photonic crystal fibre," Nature Photonics 5, 549-553 (2011).

[29] Botineau, J., Picholle, E. and Bahloul, D., "Effective stimulated brillouin gain in singlemode optical fibres," Electronics Letters 31, 2032 -2034 (1995).

[30] Wang, J., Zhu, Y., Zhang, R. and Gauthier, D. J., "Fsbs resonances observed in a standard highly nonlinear fiber," Opt. Express 19, 5339-5349 (2011).

[31] McCurdy, A. H., "Modeling of stimulated brillouin scattering in optical fibers with arbitrary radial index profile," J. Lightwave Technol. 23, 3509 (2005).

[32] Royer, D. and Dieulesaint, E., [Elastic Wvaes in Solids I: Free and Guided Propagation], Springer, (2000). 
[33] Chandorkar, S.A., Agarwal, M., Melamud, R., Candler, R.N., Goodson, K.E. and Kenny, T.W., "Limits of quality factor in bulk-mode micromechanical resonators," MEMS 2008--IEEE 21st International Conference on Micro Electro Mechanical Systems, $74-77$ (2008).

[34]Dieulesaint, E. and Royer, D., [Elastic Wvaes in Solids II: Generation, Acousto-Optic Interaction, Applications], Springer, (2000).

[35] Gordon, J. P., "Radiation forces and momenta in dielectric media," Phys. Rev. A. 8, 14-21 (Jul 1973).

[36] Johnson, S. G., Ibanescu, M., Skorobogatiy, M. A., Weisberg, O., Joannopoulos, J. D. and Fink, Y., "Perturbation theory for maxwell's equations with shifting material boundaries," Phys. Rev. E. 65, 066611 (Jun 2002).

[37] Hounsome, L. S., Jones, R., Shaw, M. J. and Briddon, P. R., "Photoelastic constants in diamond and silicon," Physica status solidi A. 203, 3088-3093 (2006).

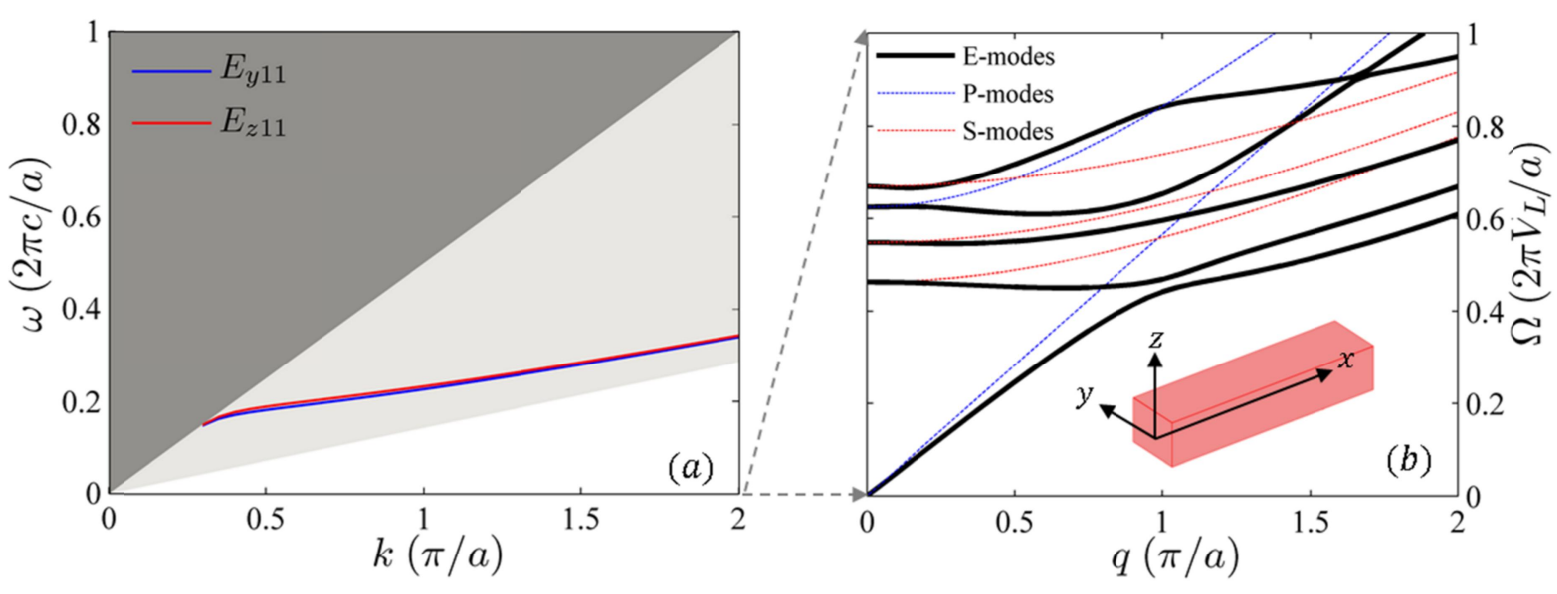

(c)

E1 (P)

E2 (S)

E3 (S)
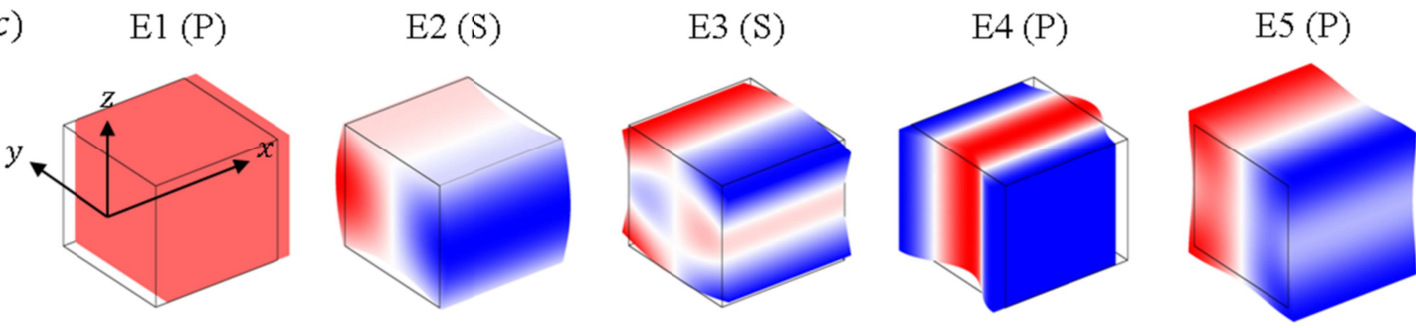

Figure 1: The optical and elastic modes of a silicon rectangular waveguide. Optical frequency is in unit of $2 \pi c / a$, while elastic frequency is in unit of $2 \pi V_{L} / a . \mathrm{V}_{\mathrm{L}}=p E / \rho=8.854 \times 10^{3} \mathrm{~m} / \mathrm{s}$ is the longitudinal sound velocity of silicon. (a) Dispersion diagram of optical modes $E_{y 11}$ and $E_{z 11}$. (b) Dispersion diagram of elastic modes which are even about both $y=0$ and $z=0$. E-modes (black lines) are the actual eigen-modes. P-modes (blue lines) are the constrained eigen-modes with only longitudinal movements. S-modes (red lines) are the constrained eigen-modes with only transverse movement. At $q=0$, Emodes are either P-modes or S-modes. (c) The modal profiles of E1 through E5 at $q=0$. The deformation is proportional to the displacement. The color represents $u_{y}$ for S-modes and $u_{x}$ for P-modes. Blue, white, and red correspond negative, zero, and positive values respectively. E1 corresponds to a longitudinal shift. 
(a)

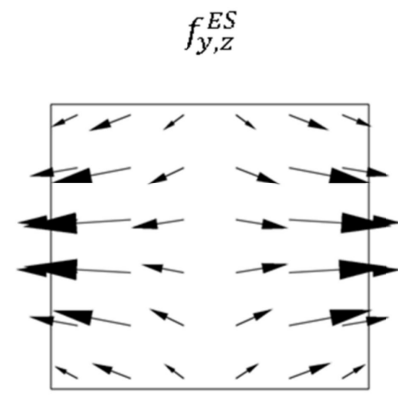

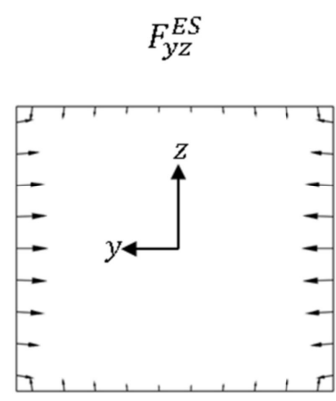
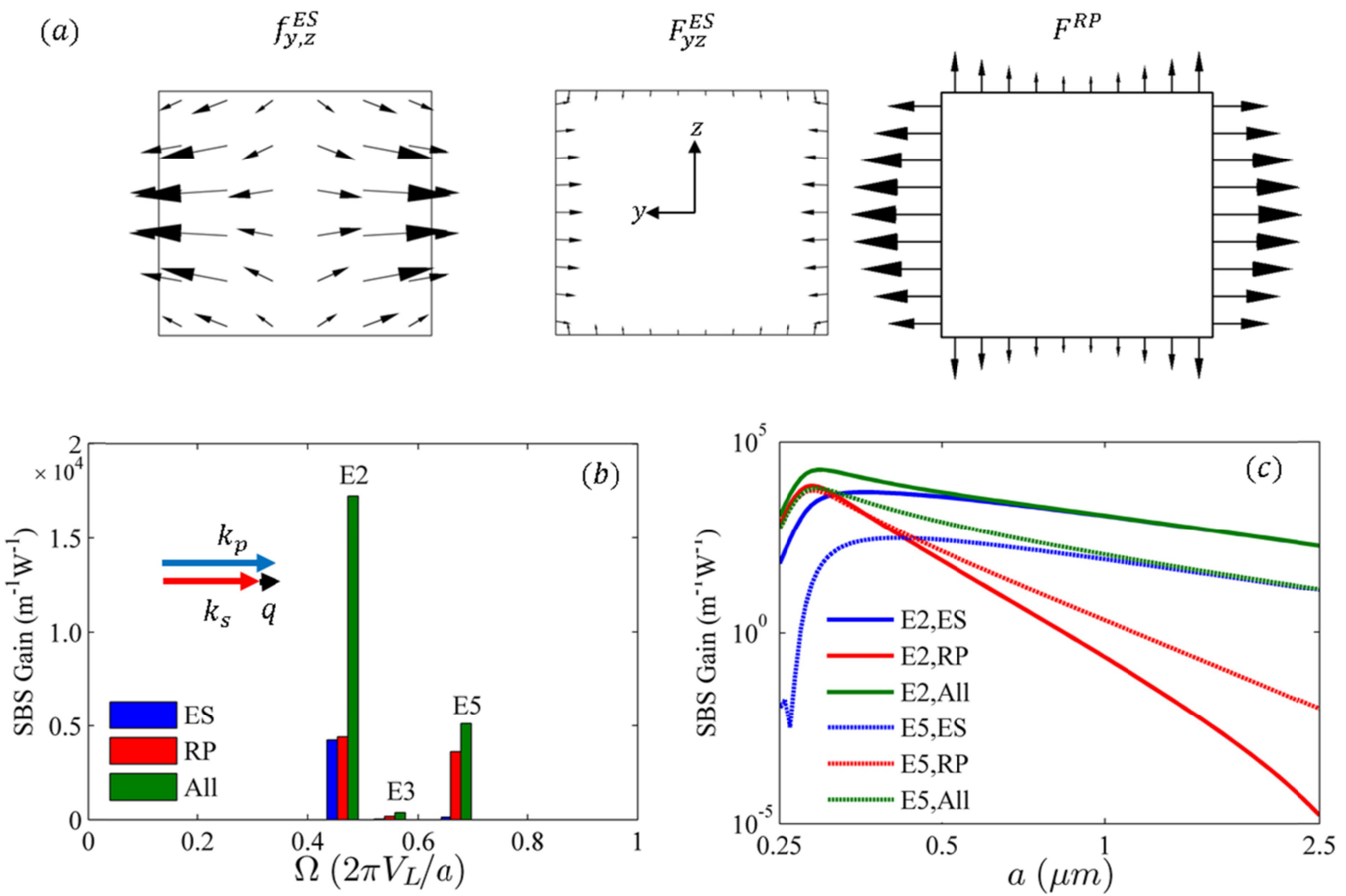

Figure 2: Detailed analysis of FSBS. For (a) and (b), the operating point is $\omega=0.203(2 \pi c / a), k=0.75(\pi / a)$, and $q=0$ with $a$ $=315 \mathrm{~nm}$. (a) The distribution of optical forces. All optical forces are transverse. (b) FSBS gains calculated from overlap integral using $Q=1000$. Blue, red, and green bars represent FSBS gains from electrostriction force, radiation pressure, and both. Only S-modes have non-zero gains. (c) The scaling of FSBS gains as $a$ varies from $0.25 \mu \mathrm{m}$ to $2.5 \mu \mathrm{m}$. Solid and dotted lines represent E2 and E5 respectively. 
(a)
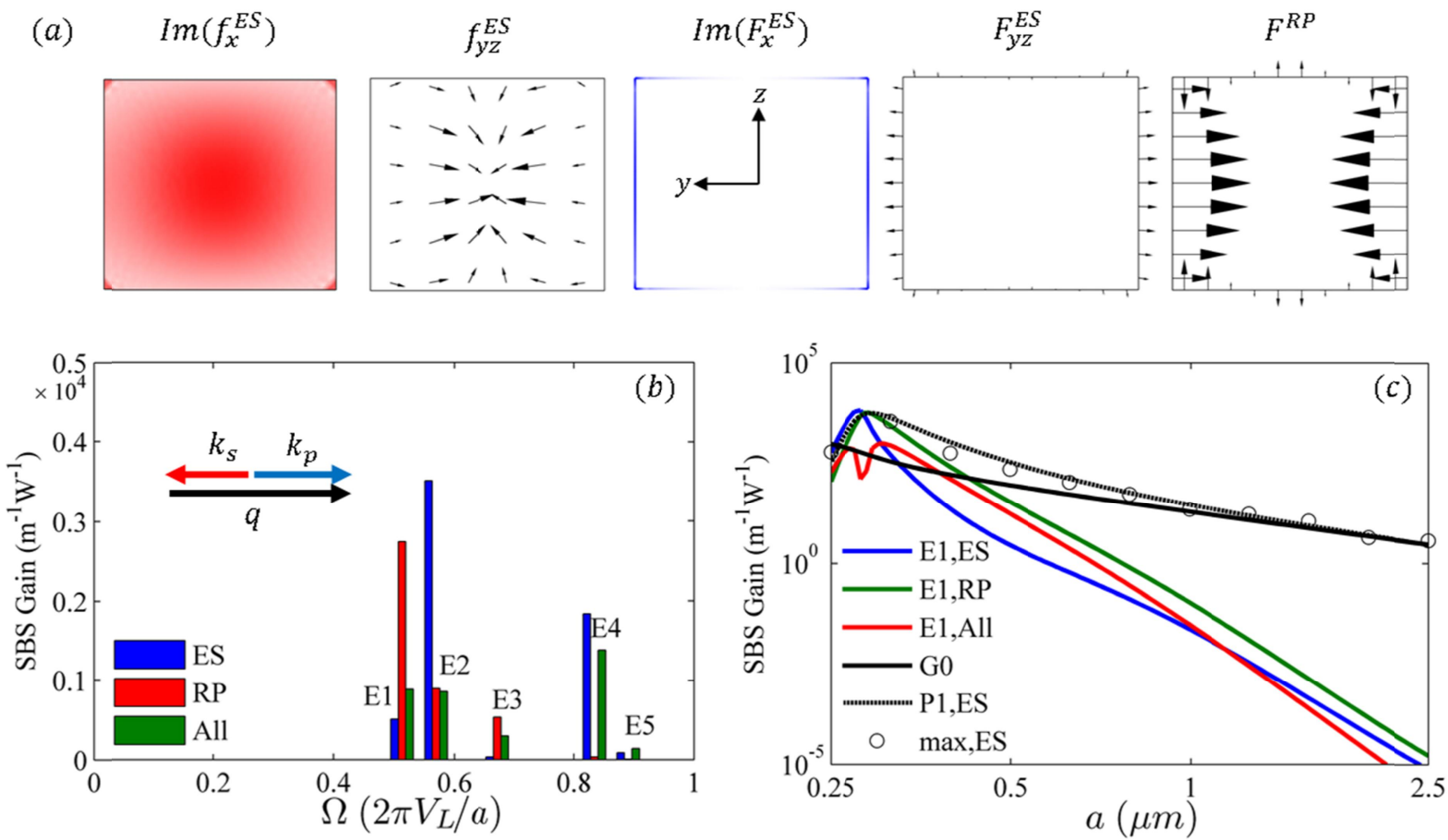

Figure 3: Detailed analysis of BSBS. For (a) and (b), the operating point is $\omega=0.203(2 \pi c / a), k=0.75(\pi / a)$, and $q=1.5(\pi / a)$ with $a=315 \mathrm{~nm}$. (a) The distribution of optical forces. Electrostriction forces have both longitudinal and transverse components. Radiation pressure has only transverse component. (b) BSBS gains calculated from overlap integral using $Q=$ 1000. Blue, red, and green bars represent FSBS gains from electrostriction force, radiation pressure, and both. (c) The scaling of BSBS gains as $a$ varies from $0.25 \mu \mathrm{m}$ to $2.5 \mu \mathrm{m}$. Blue, red, and green lines represents different BSBS gains of E1. Solid black line represents the conventional BSBS gain. Dotted black line represents the electrostriction BSBS gain of P1. Black dots represents the maximal electrostriction BSBS gain of all E-modes. 
(a) $f_{y z}^{E S}$

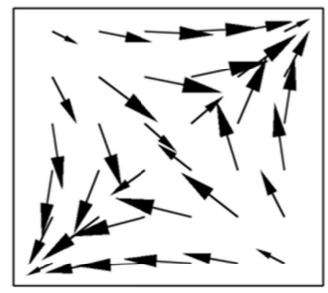

(b)
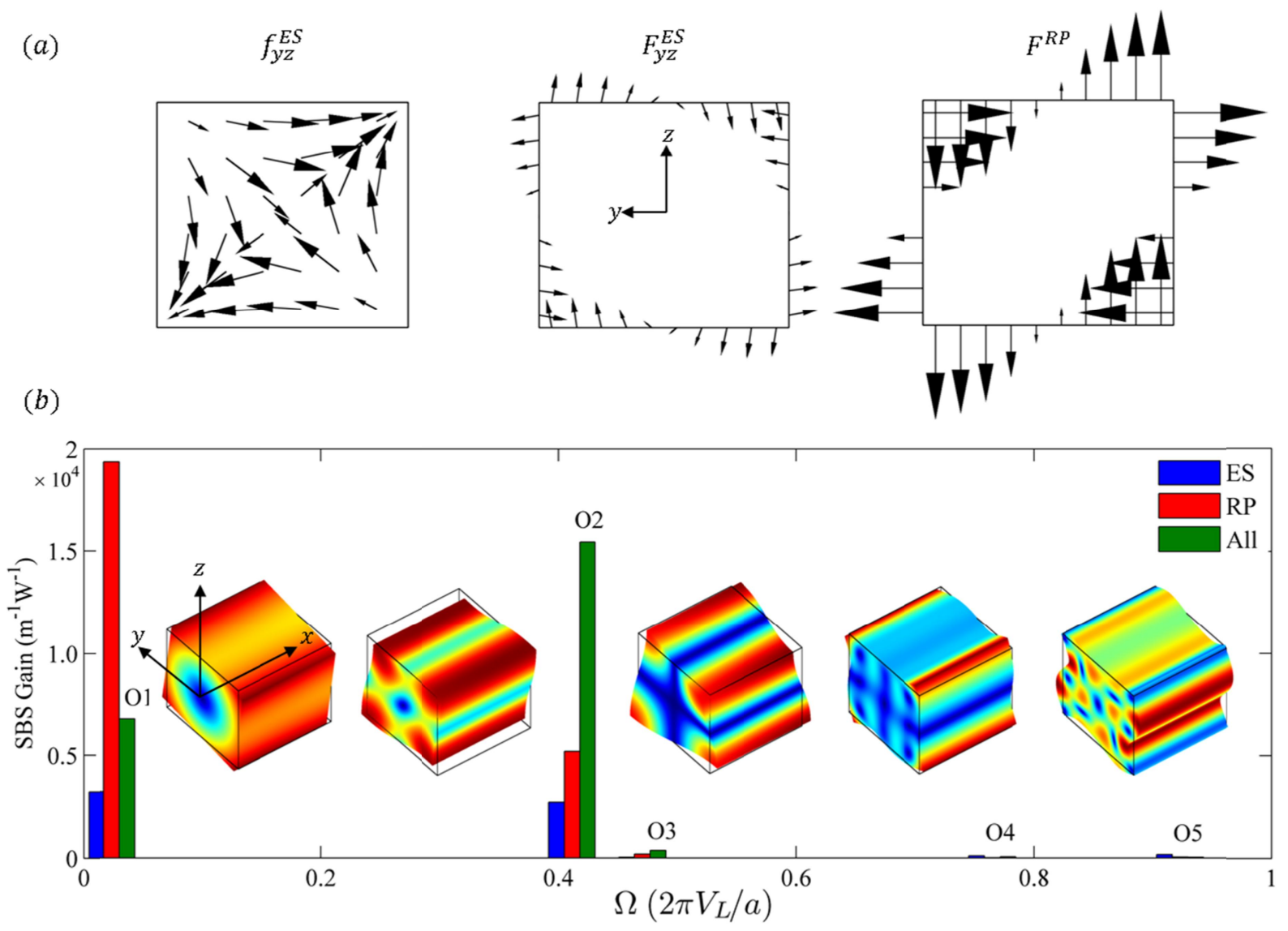

Figure 4: Detailed analysis of intermode coupling between $E_{y 11}$ (pump) and $E_{z 11}$ (Stokes). The operating point is $\omega=$ $0.203(2 \pi c / a), k_{p}=0.750(\pi / a), k_{s}=0.665(\pi / a)$, and $q=0.085(\pi / a)$ with $a=315 \mathrm{~nm}$. (a) The distribution of optical forces. The longitudinal forces (not shown here) are much smaller than the transverse forces. All optical forces are anti-symmetric about $y=0$ and $z=0$, exciting elastic modes with the same parities (O-modes). (b) Intermode SBS gains calculated from overlap integral using $Q=1000$. The model profiles of $\mathrm{O} 1$ through $\mathrm{O} 5$ at $q=0.085(\pi / a)$ are inserted. The deformation is proportional to the displacement. The color represents the amplitude of total displacement with blue and red corresponding to zero and the maximal value. 


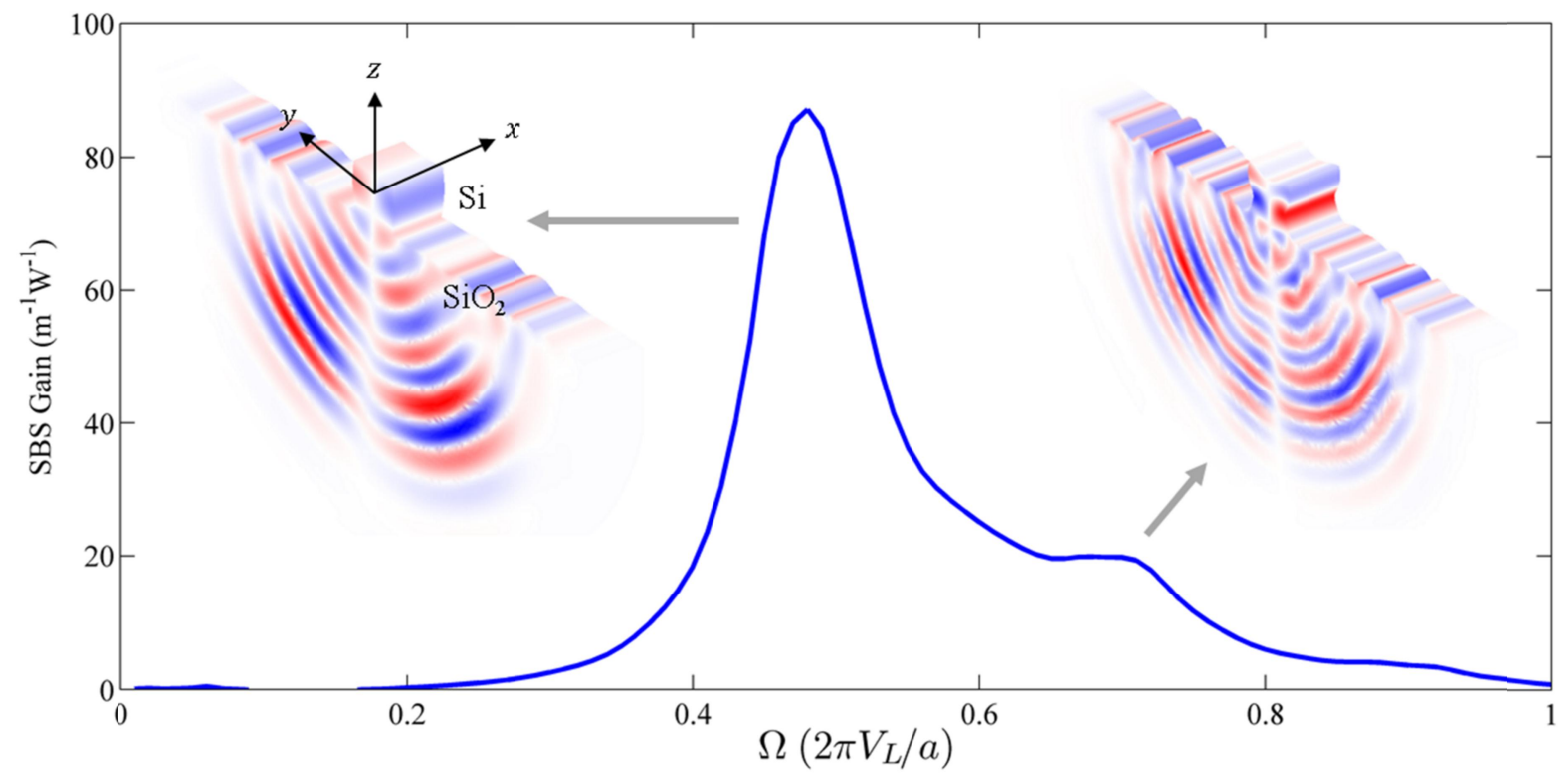

Figure 5: FSBS gains of a silicon waveguide on top of a silica substrate calculated from frequency response. For silica, we use refractive index $n=1.45$, Young's modulus $E=72.5 \times 10^{9} \mathrm{~Pa}$, Poisson's ratio $v=0.17$, and density $\rho=2200 \mathrm{~kg} / \mathrm{m}^{2}$. The operating point is $\omega=0.201(2 \pi c / a), k=0.75(\pi / a)$, and $q=0$ with $a=311 \mathrm{~nm}$. The inserts show the leaky elastic modes which give rise to the two resonant peaks. The deformation is proportional to the displacement. The color represents displacement component $u_{y}$. 